\title{
Indice delle figure
}

Figura 1 La medicina medievale fra teoria e prassi $\longrightarrow \mathbf{0 7}$ 
